\title{
Asteroseismology of the Rapidly Oscillating Ap Stars
}

\author{
L. Bigot \\ Département CASSINI - Observatoire de la Côte d'Azur \\ BP 4229 - 06304 - Nice cedex 4 - France.
}

\begin{abstract}
The pulsation properties of the rapidly oscillating Ap (roAp) stars are briefly presented. The combined effect of a strong magnetic field and rotation on pulsations is also discussed.
\end{abstract}

\section{Introduction}

It is known from the last two decades that a part of the well-known Ap stars show rapid light variations with typical periods between 5-15 minutes and with amplitudes of the order of one millimagnitude. They correspond to high order p-modes with typical number of nodes $n>20$. Since their discovery by Kurtz (1982), the number of these rapidly oscillating Ap (roAp) stars has grown up to 32 (September 2000). They have strong global dipole magnetic fields, with typical photospheric values of kilogauss strengths. In most of cases these oscillations are aligned with the magnetic field which indicates the great role played by the field on these oscillations and show that they are close to dipole modes in the magnetic reference system.

The observed spectra show multiplets. To explain this property Kurtz (1982) proposed the oblique pulsator model. In this model, the oscillations are pure dipole modes aligned with the magnetic axis which is tilted from the rotation axis by an angle $\beta$ called the obliquity. An arbitrary observer whose line of sight is inclined with rotation axis, by an angle $i$, sees a modulation of the signal. Because of this modulation, each eigenmode is seen in observer's system as a multiplet with $(2 \ell+1)$ components. This model gives then a natural explanation for the presence of multiplets, but does not explain the inequality of the peaks observed in these multiplets. Dziembowski \& Goode (1985) proposed that the modes in the magnetic frame are not pure dipole modes, because of the effect of the Coriolis force. This leads to unequal peaks in the observer's system. The magnetic field has always been considered as a small perturbation on p-modes. Dziembowski \& Goode (1996) showed that in the context of roAp stars, this cannot be true, because of the dominating magnetic pressure over the gas pressure in outer layers of the star. This effect is very important in the case of roAp stars, because they are oscillating with high orders p-modes, for which most of the amplitude is concentrated in outer layers where the magnetic field is so important. We treat the magnetic field effects with a non-perturbative approach. We also propose to include not only the effect of the Coriolis force but also the effect of the distortion of the star by the centrifugal force which is very important for roAp stars. 

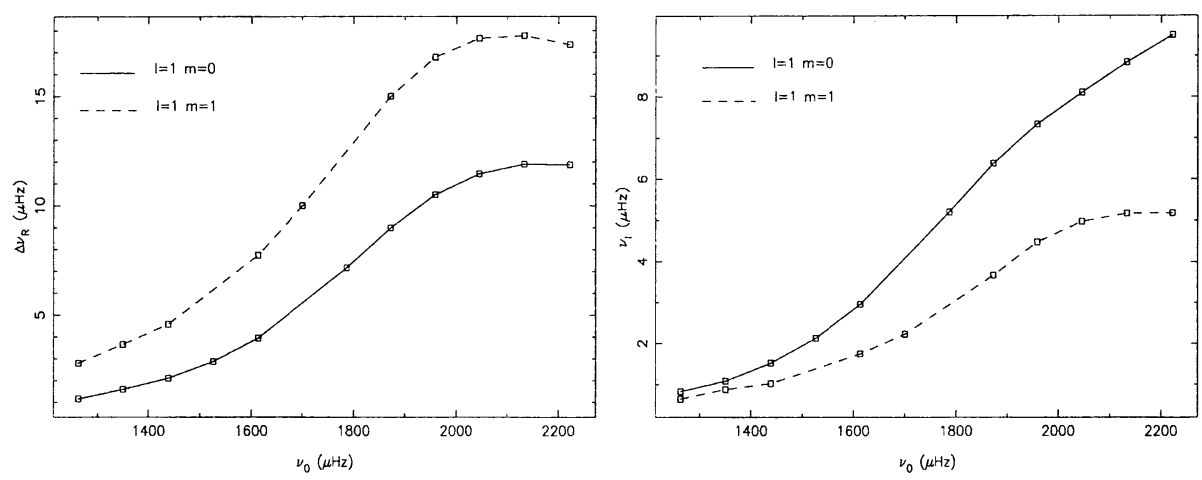

Figure 1. Plots of the real (left panel) and imaginary (right panel) shifts of the frequency for modes with $\ell=1$ and the two corresponding values of $m, m=0$ (full line) and $m=1$ (dashed line), as a function of the frequency, for one value of the magnetic field, $B=0.8 \mathrm{kG}$.

\section{Magnetic field effects}

A non-perturbative approach of the magnetic field on pulsations has been investigated in Dziembowski \& Goode (1996) for axisymmetric oscillations $(m=0)$ and generalized by Bigot et al. (2000) to non-axisymmetric oscillations $(m \neq 0)$, $m$ being the azimuthal order of the mode. They treat the magnetic field effects only in a fine layer at the top of the star where the magnetic pressure is comparable and even larger than the gas pressure, assuming a plan-parallel approximation (i.e. neglecting the horizontal derivatives of the perturbed quantities). The global eigensolutions are found matching the solutions from this thin magnetic layer with the inner non-magnetic ones. Because of the latitude dependence of the Lorentz force, the modes are distorted by the magnetic field and are represented as a linear combination involving several degrees $\ell$ 's. The effect of the magnetic field on pulsations, is to create a shift of the frequency. This shift contains real $(\Delta \omega)$ and imaginary $\left(\omega_{I}\right)$ components. This is illustrated in Fig. 1. The magnetic field raises only partially the degeneracy of the frequencies since they depend only on the absolute value of $|m|$. This is due to the fact that prograde and retrograde modes feel the same perturbation since the field is axially symmetric.The real shift of the frequency is very significant, with typical values of $10 \mu \mathrm{Hz}$, since it is of the order of the small separations and also comparable to the large separations. The imaginary shift is due to the fact that, in this magnetic layer, a part of p-mode energy is converted into Alfvénic waves which are dissipated below this layer. The complex shifts are also of the order of few $\mu \mathrm{Hz}$ but are generally smaller than the real shifts. Both of these shifts are very dependent on the magnetic strength, the number of nodes and the geometry of the mode. Moreover, it has to be note that the source of damping of the oscillations by dissipation of Alfvénic waves, does not explain the observed preference for dipole modes in the magnetic reference system. Indeed, as shown in Fig. 1, the damping rate for $m=0$ is always larger than the damping rate for $m=1$. Another mechanism is then required to explain the observed selection. 


\section{Rotational perturbation of magnetic eigenmodes}

Because of its inclined axis, the perturbation due to rotation is non-axisymmetric in the magnetic reference system. This induces a coupling of the unperturbed magnetic states of different $m$ 's and the use of a degenerated perturbation theory is needed (Dziembowski \& Goode (1985)). In this case, one must look for solutions in the form

$$
\vec{\xi}=\sum_{m=-\ell}^{\ell} \alpha_{m} \overrightarrow{\xi_{m}}
$$

with $\overrightarrow{\xi_{m}}$ the unperturbed magnetic solutions. The subscripts $n, \ell$ are omitted for clarity. We neglect two effects due to the magnetic field. We assume that the frequencies are real, and the magnetic eigenvectors are represented by a single spherical harmonic. This is true for a not too large magnetic field. It can be shown (Dziembowski \& Goode (1985)) that the equation of motion including both magnetic and rotation operators leads to the following eigensystem

$$
\sum_{m=-\ell}^{\ell} \alpha_{m}\left\{O_{j m}-\omega^{2} \delta_{j m}\right\}=0 \quad j=-\ell, \ldots, \ell
$$

with $\omega_{(s)}^{2}$ and $\alpha_{m}^{(s)}$ the $2 \ell+1$ eigenvalues and eigenvectors $(s)$ of the matrix $O_{j m}$. This matrix is the projection of the magnetic and rotation operator into the unperturbed magnetic eigenstates. Since rotation is non-axisymmetric, the matrix $O_{j m}$ is non-diagonal. The results for the frequencies are presented in Fig. 2 , for the case of $\ell=1$. The first effect of rotation is to raise the degeneracy in $|m|$ discussed in section 2 . Two of the solutions depend on the obliquity $\beta$ whereas one is almost $\beta$-independent. For roAp stars, the effect of centrifugal distortion is much larger than the Coriolis one. This is mainly due to the fact that these stars pulsate with high order p-modes. Hence, the shift created by the rotation is dominated by the contribution due to the centrifugal distortion of the star (few $\mu \mathrm{Hz}$ ). The contribution of the Coriolis force is two orders of magnitude smaller. To compare with observables, we rotate these solutions from the magnetic frame into observer's frame using the Wiener matrices, $d_{m^{\prime} m}^{(\ell)}(\lambda)$. These matrices transform spherical harmonics from one frame to another one separated by an angle $\lambda$. Then, in observer's frame the disk-average fluctuation of luminosity $L$ for each perturbed eigenmode $(s)$ writes like a multiplet with $2 \ell+1$ components separated by exactly the rotation rate $\Omega \approx$ few $\mu \mathrm{Hz}$,

$$
\left(\frac{\delta L}{L}\right) \propto \sum_{m^{\prime}=-\ell}^{\ell} A_{m^{\prime}}^{(s)} \cos \left(\omega_{(s)}-m^{\prime} \Omega\right) t \quad A_{m^{\prime}}^{(s)} \propto d_{m^{\prime} 0}^{(\ell)}(i) \sum_{m^{\prime}=-\ell}^{\ell} \alpha_{m}^{(s)} d_{m m^{\prime}}^{(\ell)}(\beta) .
$$

The amplitudes $A_{m^{\prime}}^{(s)}$ depend on several parameters of the star, like the magnetic field, the obliquity $\beta$ and the inclination $i$. Then, a comparison between observed and calculated amplitudes can provide an estimation of the magnetic field strength in roAp stars. In Fig. 2, we plot for $\ell=1$, the ratio $\gamma^{(-)}=\left(A_{1}-A_{-1}\right) /\left(A_{1}+A_{-1}\right)$ (e.g. Kurtz \& Shibahashi (1986)), for one of the three possible eigenmodes. This ratio, and then the inequality of peaks, 

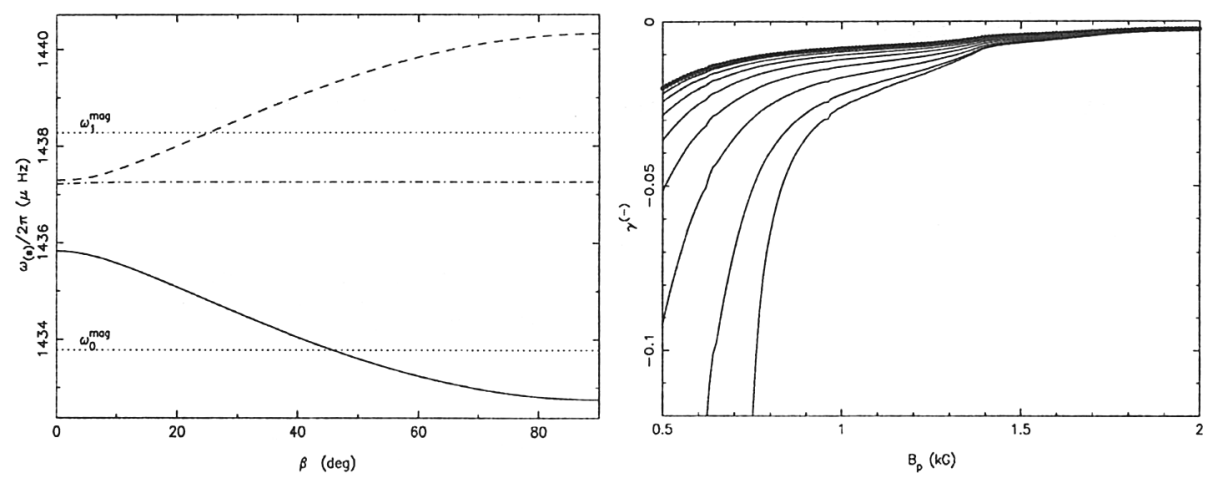

Figure 2. (Left panel) Plots of the three perturbed eigenfrequencies in the case of $n=23$ and $\ell=1$ as a function of the obliquity $\beta$ and for a given $B=1.0 \mathrm{kG}$. The dotted lines represent the unperturbed magnetic frequencies $\omega_{0,1}^{\text {mag }}$. (Right panel) Plots of the ratio $\gamma^{(-)}$as a function of $B$ and for various values of $\beta\left(10, . ., 90^{\circ}\right)$, and for the mode represented by a full line in the left panel. This ratio increases with decreasing $\beta$. The thick lines represent the solutions without centrifugal distortion. $\Omega=4 \mu \mathrm{Hz}$

increases for low values of the magnetic field, since the mode is more sensitive to the effect of rotation. It strongly depends on the obliquity. It vanishes for magnetic field values above $1.5 \mathrm{kG}$ which corresponds to negligible effect of rotation $\alpha_{m \neq 0} \rightarrow 0$ and the mode behaves like a dipole mode. The change due to centrifugal distortion compared with Coriolis force alone is very significant.

\section{Conclusion}

The roAp stars is a group of very interesting and unusual pulsating stars in which both rotation and magnetic field play a great role in their oscillations. The oblique pulsator model accounts for most of their pulsations properties and it is, so far, the best model to interpret the observations. We propose here some improvements which consist in including effect of the centrifugal distortion of the star and treating the effect of the magnetic field with a non-perturbative theory which are, in our view, very important for the predictions of this model.

\section{References}

Bigot L., Provost J., Berthomieu G., Dziembowski W. A., \& Goode P. R. 2000, A\&A, 356, 218

Dziembowski, W. A., Goode P. R. 1985, ApJ Letters, 296, L27

Dziembowski, W. A., Goode P. R. 1996, ApJ, 458, 338

Kurtz, D. W. 1982, MNRAS, 200, 807

Kurtz, D. W., Shibahashi, H. 1986, MNRAS, 223, 557 